\title{
Simulation of Flow over a Rotationally Oscillating Square Cylinder at Low Reynolds Numbers
}

\author{
N. K. Singh \\ Department of Mechanical Engineering, National Institute of Technology, \\ Kurukshetra 136119, Haryana, India \\ Email: nksinghfme@nitkkr.ac.in \\ Phone: +919671200887
}

\begin{abstract}
Simulation of uniform flow past a square cylinder undergoing rotational oscillations at various frequencies is performed using the ANSYS Fluent CFD package. The frequency ratios $F_{R}$ chosen for the study are $0.4,0.8$ and 1.0. At each frequency ratio, a number of angular amplitudes are selected, and the simulations are performed using a dynamically deforming mesh. The results are obtained from the simulations in terms of the nondimensional drag and lift forces, vorticity contours, power spectral density plots of lift coefficients and Strouhal number. A general increase in lift and drag coefficients with increasing frequency ratios as well as with increasing amplitudes at a particular frequency ratio, except a few anomalies, is seen. The flow features in the wake and the vicinity of the cylinder are compared for the various parameters. The use of convective boundary condition for the problem is demonstrated.
\end{abstract}

Keywords: Square cylinder; rotational oscillations; low Reynolds number; Strouhal number.

\section{INTRODUCTION}

Flow past a bluff body is an important phenomenon with interesting features and wideranging applications in flow control and flow-induced vibrations. However, most of the work in the past has been done on the flow past circular cylinders. This is mainly because flow past circular cylinders represents a wider variety of physical problems compared to any other bluff body. So, it is important to delineate the characteristics of square cylinders that differentiate them from circular cylinders. The most important and obvious among them is the location of the flow separation point on the surface of the cylinders. In case of circular cylinders, the location of the separation point continuously varies with the Reynolds number, moving back when the boundary layer transitions to turbulence and moving a bit forward at transcritical Reynolds number $\left(\operatorname{Re}>3.5 \times 10^{6}\right)$. Whereas, in the case of square cylinders, though it may be against our intuition, up to a Reynolds number of about 100, the separation of flow takes place at the trailing edges of the square cylinder oriented with a face perpendicular to the flow direction. At a Reynolds number of 125 , the flow mostly separates from the trailing edges but occasionally from the leading edges of the cylinder [1]; at a Reynolds number of 150, the flow separates mostly from the leading edge of the cylinder and occasionally from the trailing edge; beyond a Reynolds number of 175, the flow always separates from the leading edges of the cylinder.

Another important difference is related to the case of flow past oscillating cylinders. In the case of flow past circular cylinders oscillating linearly transverse to the 
flow, there is an increase in the non-dimensional drag acting on the cylinder; whereas for the same case considered concerning the square cylinder, there seems to be a reduction in the magnitude of non-dimensional drag. The observations get swapped when it comes to rotational oscillations - drag reduces in circular cylinders and increases in square cylinders.

In comparison to circular cylinders, investigations of the flow past a square cylinder have been reported less frequently in the literature. Some of these investigations reveal flow features some of which are unique to square cylinder case and others directly in contrast to the circular cylinder case. Vickery [2] conducted experiments in a wind tunnel to simulate the wind loads acting on buildings. He used a square cylinder, 6in. $\times$ 6in. in cross-section mounted transversely in a low-speed wind tunnel. The turbulence intensity in the wind tunnel was maintained to correspond with the atmospheric turbulence level. The experiments were conducted for Reynolds numbers ranging from 4 $\times 10^{4}$ to $1.6 \times 10^{5}$ on the square cylinder at different angles of attack $(\alpha)$. The average values of the pressure coefficients, drag coefficients, lift coefficients and Strouhal numbers were corrected for the blockage effect. It was found that the change in $\alpha$ had very little impact on Strouhal number (S). It was also reported in the investigation that the effect of free stream turbulence had very little impact on $S$ but reduced the root mean square (RMS) value of the fluctuating lift coefficient (non-dimensional lift force) $\mathrm{C}_{\mathrm{L}, \mathrm{rms}}$ to a great extent at the value of $\alpha$ less than $15^{\circ}$. The maximum reduction in $\mathrm{C}_{\mathrm{L}, \mathrm{rms}}$ was about 50\%. It was also observed that was a reduction in the RMS value of the base pressure coefficient $\mathrm{C}_{\mathrm{pb}}$ and the author hypothesised that it was due to reattachment which was possible at low values of $\alpha$.

A similar investigation was carried out by Lee [3] where the author controlled the free stream turbulence level by changing the size of the mesh grid, constructed from aluminium bars, in the low-speed wind tunnel. The author showed that an increase in the free stream turbulence intensity leads to an increase in $\mathrm{C}_{\mathrm{pb}}$ and thereby, a decrease in drag.

Many investigations have been conducted on the effect of aspect ratio on the flow past bluff bodies. One such investigation was part of the experiments performed by Delany and Sorensen [4], who presented the drag coefficients and the shedding frequencies measured in a wind tunnel, for bodies with various cross-sectional shapes. Another interesting experiment was performed on rectangular cylinders [5]. In this work, the authors discussed the entrainment process wherein the growing vortices and the shear layers draw in fluid from the base of the cylinder thereby maintaining the low-pressure condition there.

\section{Investigations on Oscillating Cylinders}

Vortex-induced vibrations were known for a long time, and vortex shedding and the subsequent fluctuations in the lift and the drag forces were known to be the causes for such vibrations. But the effects on flow features due to cylinders vibrating in the flow were not investigated until the 1970s. Taneda [6] conducted a series of experiments on the flow past rotational oscillations of circular cylinders at various Reynolds numbers and oscillation frequencies at an amplitude of $45^{\circ}$. The experiments were conducted in a range of Reynolds number $30-300$ and flow visualisation techniques were used in the investigation. He discovered a critical frequency beyond which the wake region behind the cylinder disappears, and the flow pattern seems to appear like that of a potential flow although the flow itself cannot become one. Investigators in later years conducted similar 
experiments on flow past rotationally oscillating circular cylinders at higher Reynolds numbers and using other techniques such as numerical modelling.

Bearman and Obasaju [7] conducted experiments on the flow past transverse oscillations of square cylinders at various amplitudes in terms of cylinder width and various frequencies in terms of reduced velocity $\mathrm{U}_{o} / \mathrm{ND}$, where $\mathrm{N}$ is the frequency of oscillation, $\mathrm{U}_{\infty}$ is the free stream velocity and $\mathrm{D}$, the width of the square cross-section. The Reynolds number range considered for the study was $5.8 \times 10^{3}$ to $3.2 \times 10^{4}$. Important observations reported by the author were the reduction in correlation length with increasing Reynolds numbers and the reduction in base suction coefficient $\left(-\mathrm{C}_{\mathrm{pb}}\right)$ and hence drag reduction. The reason for the reduction in drag coefficient was explained in terms of the amount of circulation that flows from the shear layers to the vortices, mainly deriving from the work of Roshko [8]. Impact of varying the amplitude and frequency of the oscillation and the Reynolds number of the incoming flow was examined in depth by Leontini et al. [9]. Recently Zhao et al. [10] experimentally investigated the in-line flowinduced vibration (FIV) of an elastically mounted circular cylinder under forced axial rotation in a free stream.

A cylinder can rotationally oscillate in two ways, either about its axis or in-line or transverse. Study of rotational oscillation of a cylinder about its axis has not received as much attention as in-line or transverse oscillations [11]. Further, to the best of this author's knowledge, no such study has been conducted on rotational oscillations of square cylinders. This study is an attempt to fill this gap to some extent.

\section{METHOD}

\section{Governing Equations}

At the Reynolds number considered in the present case $(\operatorname{Re}=100)$, the flow is two dimensional, and for the velocity considered, the flow remains incompressible. Hence the governing equations can be simplified based on these assumptions. The simplified continuity equation in two-dimensional Cartesian coordinates can be given as,

$$
\frac{\partial \mathrm{u}}{\partial \mathrm{x}}+\frac{\partial \mathrm{v}}{\partial \mathrm{y}}=0
$$

Where, $\mathrm{u}$ and $\mathrm{v}$ are the velocity components in $\mathrm{x}$ and $\mathrm{y}$ directions respectively. The other two equations that govern the present fluid flow problem are the momentum conservation equations which can be given as:

x-momentum equation:

$$
\frac{\partial u}{\partial t}+u \frac{\partial u}{\partial x}+v \frac{\partial u}{\partial y}=-\frac{1}{\rho} \frac{\partial p}{\partial x}+v\left(\frac{\partial^{2} u}{\partial x^{2}}+\frac{\partial^{2} u}{\partial y^{2}}\right)
$$

y-momentum equation:

$$
\frac{\partial \mathrm{v}}{\partial \mathrm{t}}+\mathrm{u} \frac{\partial \mathrm{v}}{\partial \mathrm{x}}+\mathrm{v} \frac{\partial \mathrm{v}}{\partial \mathrm{y}}=-\frac{1}{\rho} \frac{\partial \mathrm{p}}{\partial \mathrm{y}}+\mathrm{v}\left(\frac{\partial^{2} \mathrm{v}}{\partial \mathrm{x}^{2}}+\frac{\partial^{2} \mathrm{v}}{\partial \mathrm{y}^{2}}\right)
$$




\section{Computational Domain}

The size of the domain that is considered for the numerical simulation should be large enough as it will be simulating a flow which is not bounded by walls. There have been investigations in the past that studied the blockage effect of the bounding walls by varying a parameter called as blockage ratio $(\beta)$ where $\beta$ is the ratio of the characteristic dimension of the cylinder to the height of the domain, i.e., as the value of $\beta$ increases, the influence of the walls on the flow variables increases. In the present work the dimensions of the domain, as shown in Figure 1, have been varied to study their effects on the integral quantities such as time histories of drag and lift coefficients.

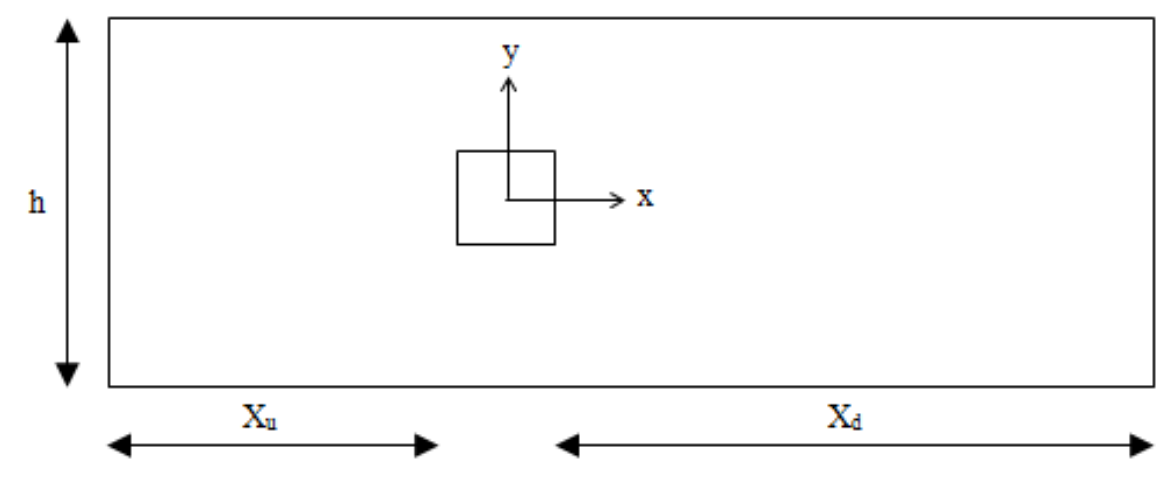

Figure 1. Computational domain dimensions.

In Figure 1, the notations ' $h$ ', ' $X_{u}$ ' and ' $X_{d}$ ' stand for the height of the domain, upstream or approach dimension and the downstream dimension respectively. All these dimensions are usually reported in terms of a number of units of a characteristic dimension of the cylinder, i.e., all the dimensions above are made non-dimensional concerning the cylinder dimension which in this case is the side length of the cylinder cross-section.

The simulations for these preliminary analyses were done considering the cylinder stationary and the flow uniform around the cylinder at a Reynolds number of 100. The discretisation and the solution schemes utilised for these simulations were the ones used for the actual problem simulations and will be explained in the sections that follow. The comparisons of $C_{D}$ and $C_{L}$ time histories for the various cases are shown in Figure 2 . The mean numbers obtained from these plots were compared with the results of the numerical investigations carried out by Sohankar et al. [12], and the comparisons are listed in Table 1. 


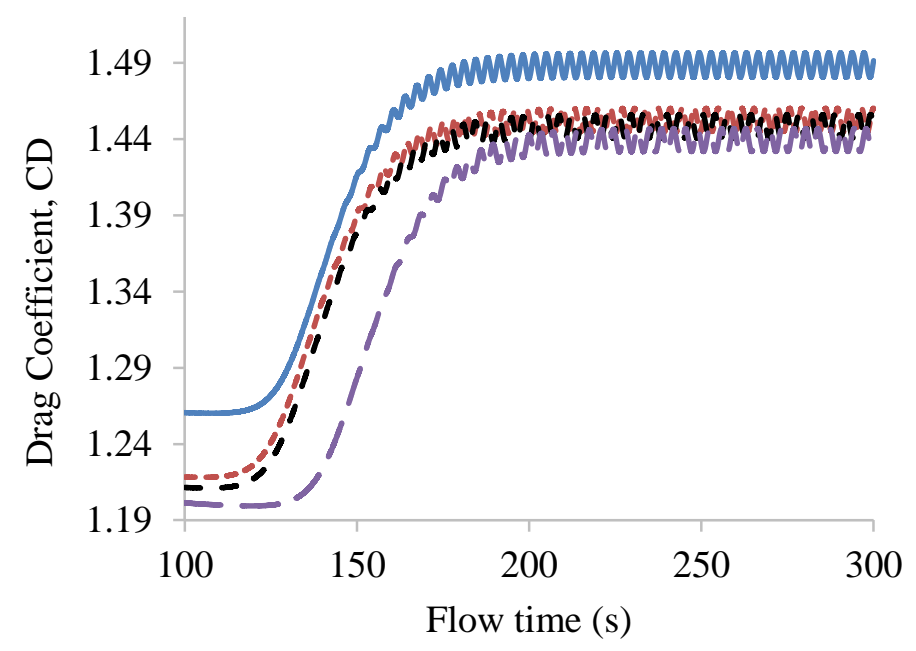

(a)

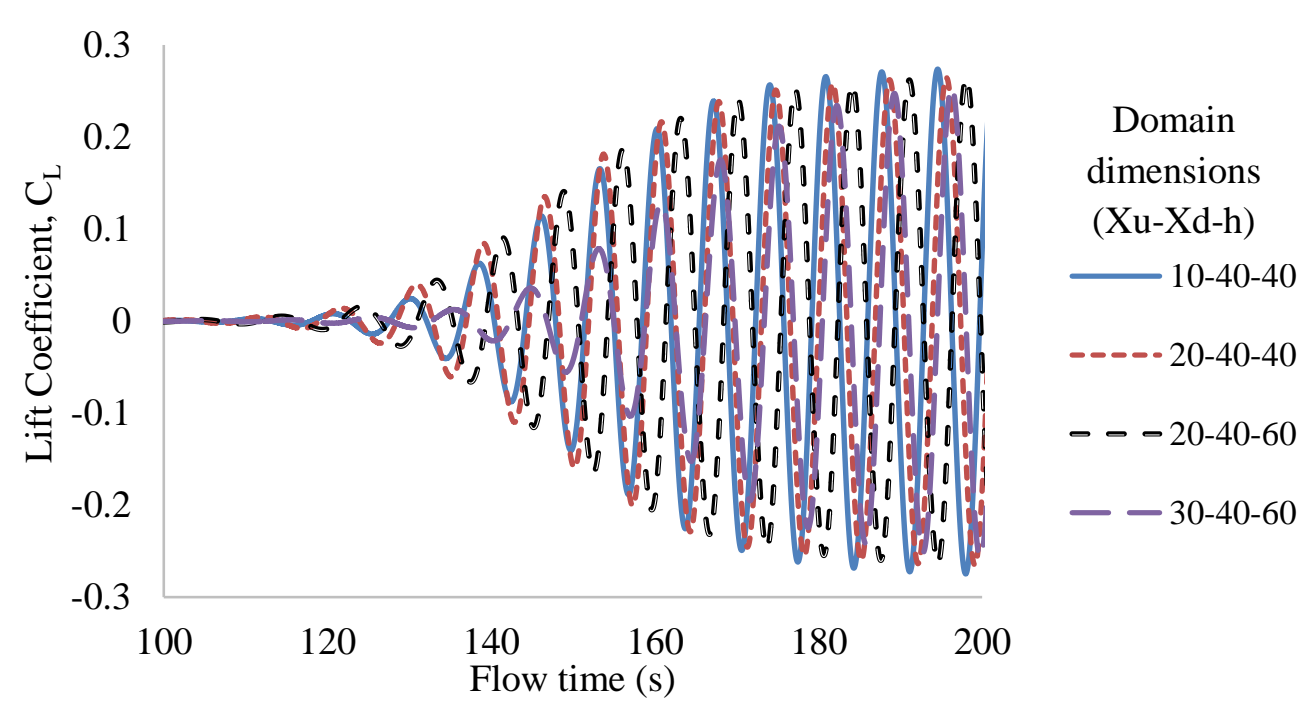

(b)

Figure 2. Effect of the computational domain dimensions on the time histories of (a) drag coefficient and; (b) lift coefficient.

Table 1. Mean values of the integral quantities from preliminary analyses.

\begin{tabular}{|c|c|c|c|c|c|}
\hline Sr. no. & $\begin{array}{c}\text { Domain size } \\
\left(\mathrm{X}_{\mathrm{u}}, \mathrm{X}_{\mathrm{d}}, \mathrm{h}\right)\end{array}$ & $\begin{array}{c}\text { No. of cells } \\
\text { (quad elements) }\end{array}$ & $\mathrm{C}_{\mathrm{D} \text {,mean }}$ & $\mathrm{C}_{\mathrm{L}, \mathrm{rms}}$ & $\mathrm{S}$ \\
\hline $1[11]$ & $X_{d}=10,26$ & - & 1.464 & 0.138 & 0.146 \\
\hline 2 & $10,40,40$ & 40500 & 1.4884 & 0.1964 & 0.1457 \\
\hline 3 & $\overline{0,40,}$ & 43500 & 1.4525 & 0.1887 & 0.1449 \\
\hline 4 & $-\overline{0,40, v v}$ & 59100 & 1.4483 & 0.1878 & 0.1443 \\
\hline 5 & $30,40,60$ & 61200 & 1.4393 & 0.1867 & 0.1432 \\
\hline
\end{tabular}

From the drag coefficient plot, the mean value of the drag coefficient was obtained after the flow had attained a periodic state. In the case of the lift coefficient, as can be seen in Figure 2 (b), the mean value of the lift coefficient is zero. Hence as per convention, 
the root means square (RMS) of the lift coefficient was estimated from the lift coefficient plot after the flow had reached the periodic state. It was found that the RMS value of the lift coefficient was persistently higher than that reported in the literature. It was initially thought that the choice of BCs at the domain walls affected this result. To clarify this, simulations were done with circular domain since they obviate the need for the domain walls. The value of the RMS lift coefficient obtained from those simulations matched with those reported in the table and hence were also higher than the values observed in the literature. From the plots and the table, the domains that gave closer results seemed to be those with the configurations 20,40, 40 and 20,40, 60. Since the actual simulation was to be performed on an oscillating cylinder, the domain with the greater height -60 times the cylinder width was chosen for all the simulations to be explained about in the following sections.

\section{Boundary Conditions}

In the preliminary analyses along with the dimensions of the domain, a number of different types of boundary conditions were experimented with to study their effects on the integral parameters. Since the present investigation is on uniform flow, 'velocity inlet' boundary condition was used at the inlet with the y-component of the velocity assigned zero and the $\mathrm{x}$-component assigned a constant value; the static pressure at the inlet was assumed atmospheric. For convenience, the constant value of $\mathrm{x}$-component was assumed as $1 \mathrm{~ms}^{-1}$ just as the cylinder width was assumed as 1 unit. Based on these assumptions, the viscosity of the fluid was varied to match the required Reynolds number, in this case, a value of Re 100. The boundary conditions at inlet represent the free stream condition of the fluid flow and were utilised in the estimation of integral quantities. The lateral walls of the domain should be specified as slip walls as the walls should not interfere with the flow by inducing shear. The 'symmetry' BC available in ANSYS Fluent can be utilised to invoke free-slip BC at the walls. The application of symmetry BC implies that the normal gradients of all variables and the normal component of velocity become zero at the walls, i.e., for this simulation,

$$
\mathrm{v}=0, \frac{\partial \mathrm{u}}{\partial \mathrm{y}}=0
$$

The free-slip BC can also be applied in ANSYS Fluent by first invoking the 'wall' boundary condition at the walls and then specifying the shear stress value as zero. Specifying the free stream conditions at the lateral walls is not uncommon either; this is especially the case when C-type grid is used instead of a rectangular domain, where in a semicircle forms the front portion of the computational domain. C-grid is usually used for the 2D simulation of flow over airfoils. In the present investigation 'symmetry' BC is used at the walls for all the simulations. The surface of the cylinder was specified with the no-slip boundary condition. As mentioned earlier, for the preliminary analyses the cylinder was assumed to remain stationary, but for the simulations of the actual problem, a sinusoidal rotational oscillation was given to the cylinder surface in ANSYS Fluent using a user-defined function (UDF) written in C language and compiled using the 'Microsoft Visual Studio Express 2012'. The computational domain with the boundary conditions discussed above is shown in Figure 3. 


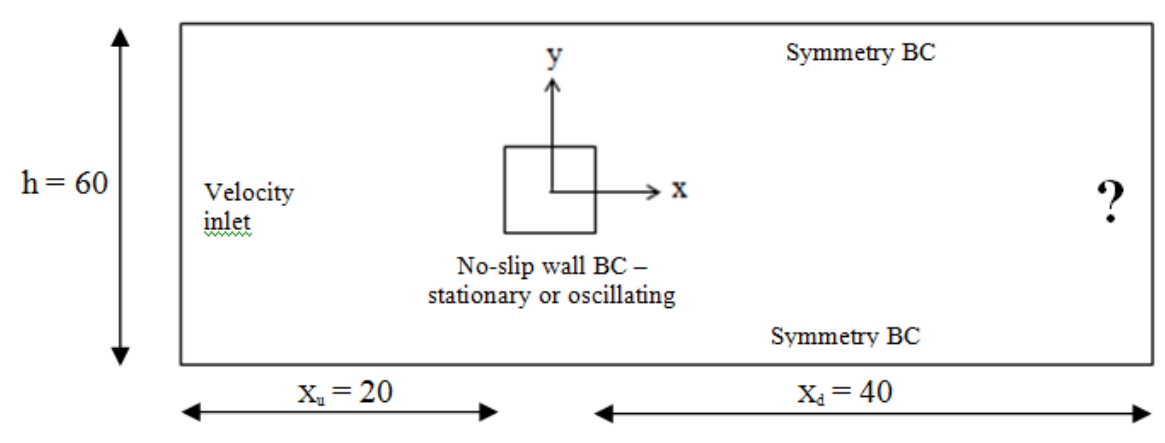

Figure 3. Computational domain with boundary conditions.

The choice of boundary condition at the outlet of the domain is of great importance. A variety of boundary conditions are available to be chosen from, such as 'pressure outlet', 'outflow BC or the Neumann BC', 'convective BC or the non-reflecting boundary condition'. A suitable boundary condition at the outlet boundary should allow the flow structures generated within the domain to freely pass through the outlet without undergoing significant distortion [13]; such a boundary condition is termed as an 'open' boundary condition. One such boundary condition namely the convective boundary condition (CBC) was given by Orlanski [13] and can be stated as given by Eq. (5).

$$
\frac{\partial \mathrm{u}}{\partial \mathrm{t}}+\mathrm{U}_{\mathrm{c}} \frac{\partial \mathrm{u}}{\partial \mathrm{x}}=0
$$

$\mathrm{U}_{\mathrm{c}}$ represents the convective velocity of the vortices leaving the outlet of the domain. It was suggested in [12] that the free stream velocity $U_{\infty}$ be taken as the convective velocity $\mathrm{U}_{\mathrm{c}}$. The discretised form of the above equation as given in [12] is:

$$
\begin{aligned}
& \mathrm{U}_{\mathrm{i}}^{*}=\mathrm{U}_{\mathrm{i}}^{\mathrm{n}}-\frac{\Delta \mathrm{t}}{(\Delta \mathrm{x})_{\mathrm{i}}} \mathrm{U}_{\mathrm{c}}\left(\mathrm{U}_{\mathrm{i}}^{\mathrm{n}}-\mathrm{U}_{\mathrm{i}-1}^{\mathrm{n}}\right) \\
& \mathrm{U}_{\mathrm{i}}^{\mathrm{n}+1}=\mathrm{U}_{\mathrm{cor}}+\mathrm{U}_{\mathrm{i}}^{*}
\end{aligned}
$$

$\mathrm{U}_{\text {cor }}$ is the correction velocity that can compensate for the difference between the mass flow at the inlet and the outlet, calculated at the end of each iteration.

\section{Solution Procedure}

With the computational grid available, it is then necessary to obtain the algebraic equations of the flow variables at all the grid points. The algebraic equations can be obtained by discretising the governing partial differential equations given by Eq. (1), (2) and (3). ANSYS Fluent uses the Finite Volume Method ( $F V M$ ) to discretise the governing equations; in FVM, the differential equations are integrated over each cell or rather, the integral forms of the governing equations are applied to each cell or finite volume.

In the discretisation of the momentum equation terms, there will be a necessity to calculate the derivatives of the velocity components. This necessitates the estimation of gradients and ANSYS Fluent provides three techniques - Least squares cell-based, Green-Gauss cell-based and Green-Gauss node-based. Among these, the Least squares 
cell-based and the Green-Gauss node-based are more accurate but expensive [14]. In this study, the Least squares cell-based technique was utilised. ANSYS Fluent uses a colocated scheme in which both the velocity scalars and pressure are stored at the cell centres [14]. However, the discretised momentum equations contain multiples of pressure and face area at all the faces of a cell. Hence, we need the pressure values at the face centres which can be obtained from interpolation of the cell centre values.

In this study, the second order interpolation scheme is used for the pressure interpolation. In this scheme, a central differencing method is used for approximating the pressure value at the face centres. For discretising the convective terms, we need the velocity values at the face centres. As already mentioned, ANSYS Fluent stores the pressure and velocity values at the cell centres. Hence the face centre values must be approximated from the cell centre values. ANSYS Fluent uses the 'upwinding' technique to estimate the values of the convective terms. In upwind schemes, the value at a face is calculated from values at a cell present upstream of the face. The upwind schemes available in ANSYS Fluent are first-order upwind, second-order upwind, power law and QUICK (Quadratic Upstream Interpolation Convective Kinetics) [15]. In this study, the second-order upwind scheme is chosen. Further, the unsteady terms are discretised using the second order implicit formulation.

As mentioned earlier, the pressure-based solver is used for this investigation. Pressure-based solution methods are further divided into segregated and coupled algorithms. In the segregated algorithms, the momentum equation is first solved followed by the solution to the pressure-correction equation. Whereas, in the coupled algorithms, both the equations are solved simultaneously. Some of the segregated algorithm techniques are SIMPLE, SIMPLEC and PISO. In this investigation, the SIMPLE algorithm was used. SIMPLE algorithm is based on the pressure correction method where a guessed pressure field is used in the momentum equation which yields values for the face fluxes. The obtained values are added with the correction face fluxes which are used to solve the continuity equation. In SIMPLE algorithm, the correction face flux is expressed as a multiple of the difference in correction pressure between the cells on the two sides of the face, and a function of the momentum equation coefficients.

\section{Solution initialization and monitors}

Apart from the residuals of continuity, $\mathrm{x}$ and $\mathrm{y}$ momentum equations, the time histories of drag and lift coefficients were monitored for the onset of periodicity in the flow calculations and these time history plots will also be used for explaining the results of the simulation in the next chapter. The time step used for the simulation could be obtained from the Courant-Lewy-Friedrich (CFL) condition if the solution techniques were of the explicit type. But in the present case, the time step size was chosen as $0.01 \mathrm{~s}$ based on the values found in the literature. The maximum number of iterations per time step was limited to 30 iterations to reduce round-off errors. The solution was initialised based on the values provided at the inlet for the uniform velocity boundary condition. The results of the simulations are presented in the next section. 


\section{RESULTS AND DISCUSSION}

\section{Drag and Lift Coefficients}

The simulations were performed at three frequency ratios $F_{R}=0.4,0.8$ and 1 and for each frequency, the maximum amplitudes $\theta_{0}$ were taken as $10^{\circ}, 20^{\circ}$ and $45^{\circ}$ respectively. For proper deformation of the dynamic mesh, the computational grid was kept coarser than that used for the study of the domain dimensions. The total number of elements used were 12108. A simulation with the coarser grid and the dynamic mode switched off was performed to derive the non-dimensional quantities. Their comparison with the finer grid and with the results of Sohankar et al. [12] is presented in Table 2.

Table 2. Comparison of the fine grid with coarser dynamic grid

\begin{tabular}{lccccc}
\hline Sr. no. & $\begin{array}{c}\text { Domain size } \\
\left(\mathrm{X}_{\mathrm{u}}, \mathrm{X}_{\mathrm{d}}, \mathrm{h}\right)\end{array}$ & $\begin{array}{c}\text { No. of cells } \\
(\text { quad elements })\end{array}$ & $\mathrm{C}_{\mathrm{D} \text {,mean }}$ & $\mathrm{C}_{\mathrm{L}, \mathrm{rms}}$ & $\mathrm{S}$ \\
\hline $1[12]$ & $\mathrm{X}_{\mathrm{d}}=10,26$ & - & 1.464 & 0.138 & 0.146 \\
\hline 2 & $20,40,60$ & 59100 & 1.4483 & 0.1878 & 0.1443 \\
\hline 3 & $20,40,60$ & 12108 & 1.4815 & 0.2001 & 0.1386 \\
\hline
\end{tabular}

From Table 2 the Strouhal number with grid available for simulation is 0.1386 . But the value reported in the literature is 0.146 . The frequency ratios $F_{R}$ used in this simulation were based on the value of $S=0.146$, or rather the natural shedding frequency 0.146 . Hence the oscillation frequencies used in this study will be a little more than $F_{R}$ times 0.1386 . For example, for the frequency ratio of 0.4 , the oscillation frequency should be 0.4 times 0.1386 which is equal to $0.0554 \mathrm{~Hz}$. But the oscillation frequency used in the simulation was 0.4 times 0.146 , which is equal to $0.0584 \mathrm{~Hz}$. This was done to check the power spectral density of the natural frequency of 0.1386 concerning that of the peak frequency at lock-in. Before describing the variation in the flow features with different frequency ratios and amplitudes, it will be useful to take a look at the variation of the nondimensional variables namely mean drag coefficient $C_{D \text {,mean }}$ and $C_{L, r m s}$, with an increasing amplitude at different frequency ratios. These are shown below in Figure 4(a) and Figure 4(b).

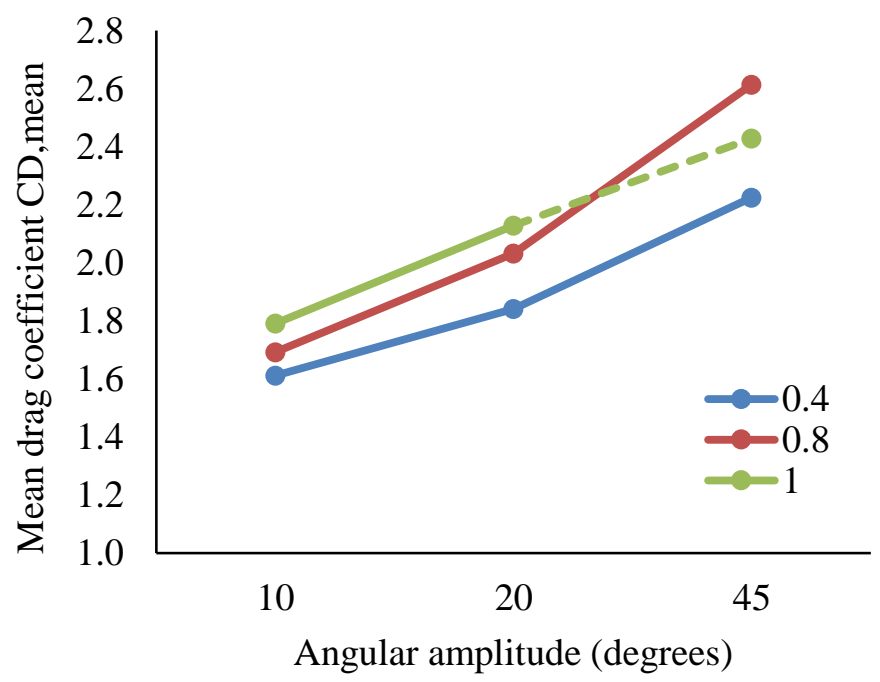

(a) 


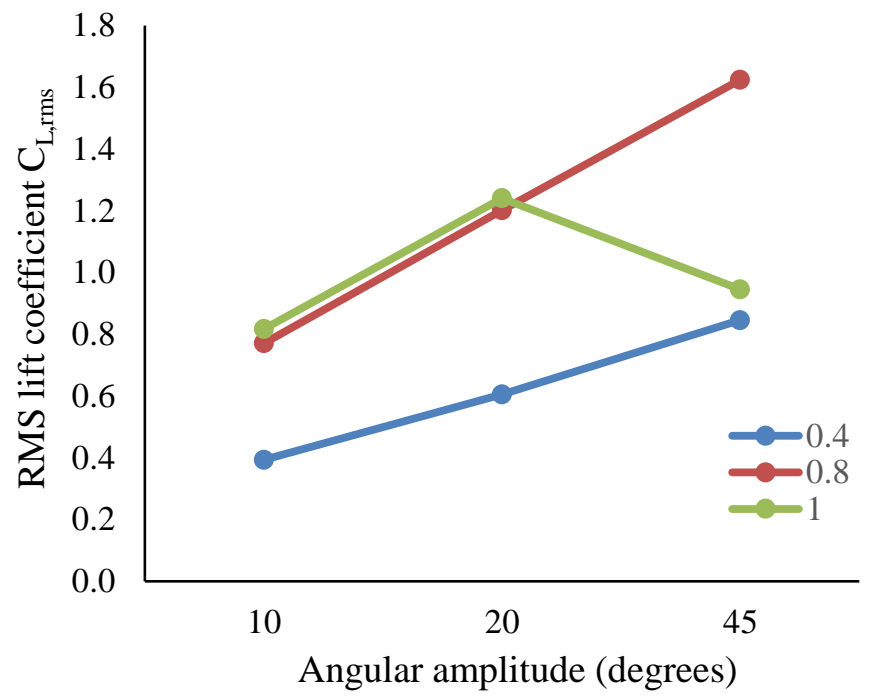

(b)

Figure 4. Variation of (a) mean drag coefficient and; (b) RMS lift coefficient with angular amplitude at various frequency ratios.

In Figure 4(a), the curve corresponding to $F_{R}=1.0$ is part shown with a dashed line. This is because for this frequency ratio at angular amplitude $\theta_{0}=45^{\circ}$, the mean drag itself varies with time a huge range of almost 1 . Hence it would not be appropriate to interpret the reduction in mean drag compared to that at $F_{R}=0.8$, as an overall reduction in drag on the body. However, in the case of RMS lift coefficient, the trend shown in Figure 4(b), directly corresponds to a decrease in RMS lift coefficient. This is because, for $F_{R}=1.0$, at $\theta_{0}=45^{\circ}$, not only does the lift coefficient periodically oscillate with time, but the amplitude of fluctuation of lift coefficient itself becomes periodic. The lift coefficient and drag coefficient plots for $\mathrm{F}_{\mathrm{R}} 0.8$ and 1.0 at $\theta_{0}=45^{\circ}$ are compared in Figure 5, (a) and (b) respectively.

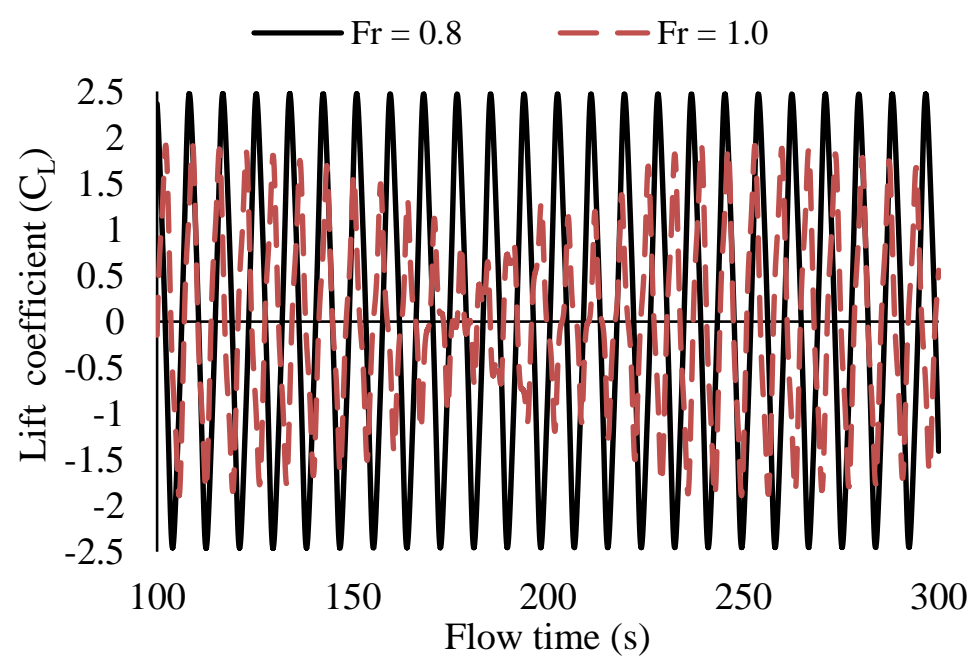

(a) 


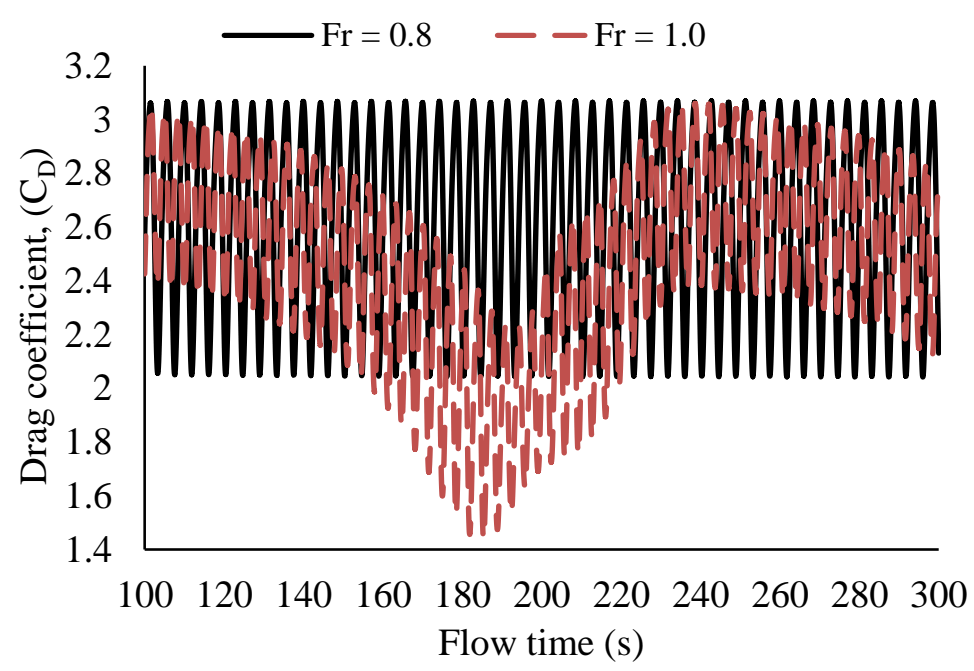

(b)

Figure 5. Comparisons of lift and drag coefficient plots for $F_{R}=0.8$ and 1 at $\theta_{0}=45^{\circ}$;

(a) lift coefficient, (b) drag coefficient.

Whether any further increase in angular amplitude at $F_{R}=1$ led to further reduction in drag coefficient was not investigated. A comparison is also required to study the variation of the effect of angular amplitude on the drag coefficient history as the frequency ratio is increased. The comparison is shown in Figure 6.

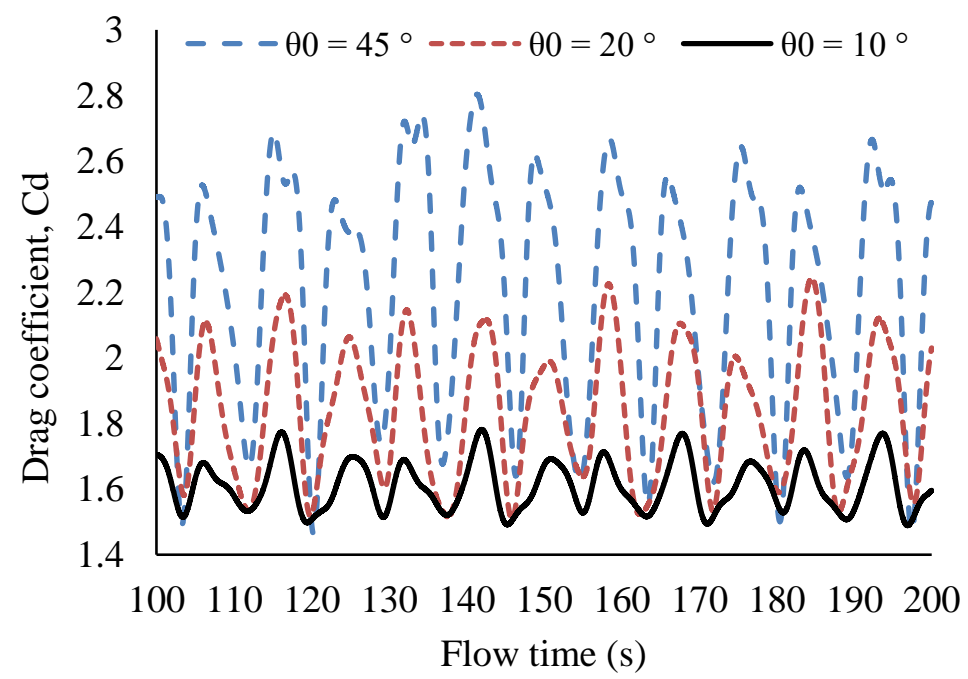

(a) $F_{R}=0.4$ 


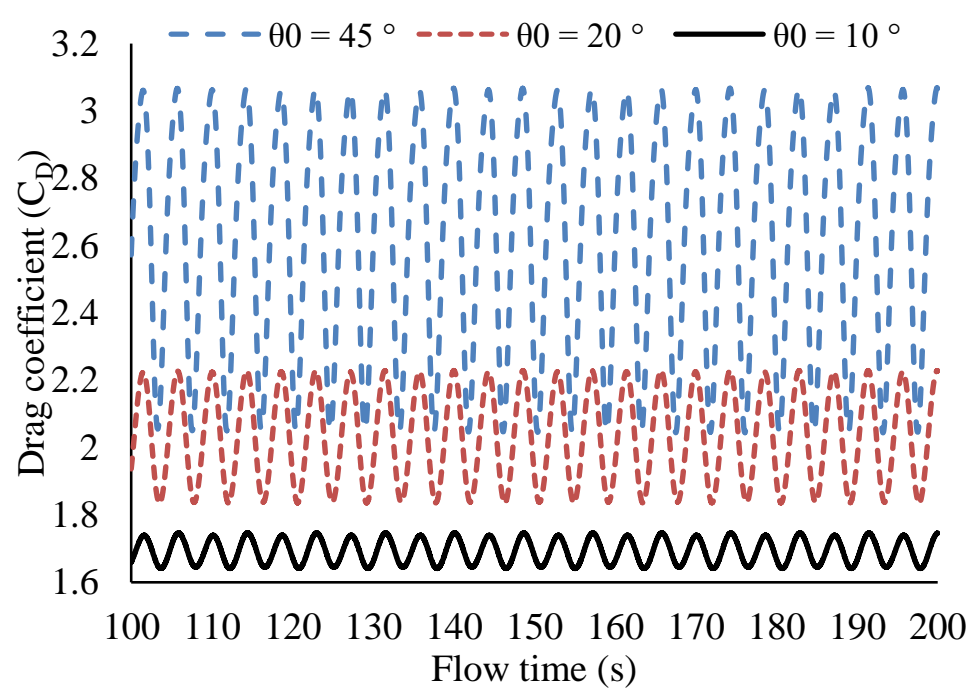

(b) $F_{R}=0.8$

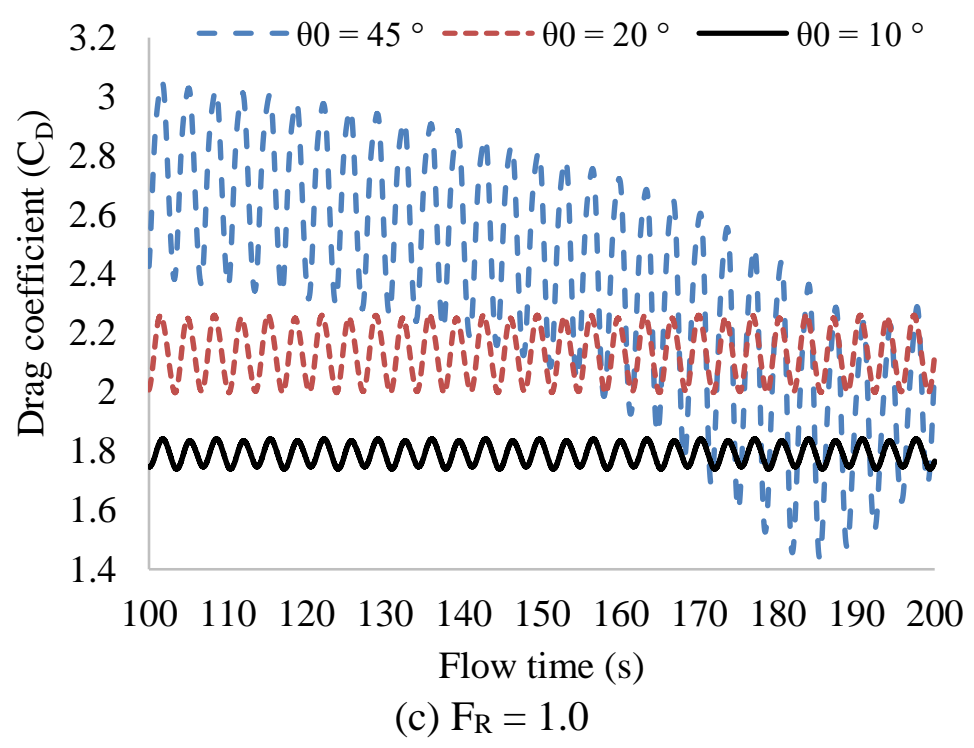

Figure 6. Effect of angular amplitude on the drag coefficient at various frequency ratios.

As can be seen in Figure 6, at a frequency ratio of 0.4, the minima on the drag coefficient plots are nearly same for different angular amplitudes although the mean $C_{D}$ values are different as already seen in Figure 4(a). As the frequency ratio is increased, not only does the mean values of $C_{D}$ move away from each other, but also the plots themselves are separated further. From this, it can be inferred that the increase in angular amplitude has a greater effect on the drag coefficient at higher frequency ratios or rather, higher oscillation frequencies. Another feature which distinguishes the plots in Figure 6, is that at $F_{R}=0.4$, the $C_{D}$ has more local fluctuations, especially at $\theta_{0}=45^{\circ}$, as compared to the smooth periodic curves at frequency ratios 0.8 and 1.0. At $F_{R}=1.0$, though the amplitudes of fluctuations are themselves time-periodic, there are no local fluctuations. This is true of the lift coefficient curves too. This can be explained in terms of the spectral density of power or power spectral density (PSD) as it is commonly known. This will be discussed in the next section to explain the more important lock-in phenomenon. 


\section{Lock-in Phenomenon and the Strouhal Number}

When the cylinder oscillates at a frequency beyond a threshold value, the vortex shedding process of the cylinder moves from its natural frequency defined by Strouhal number S, to the frequency of oscillation. This phenomenon is termed as lock-in and does not occur at a unique value but happens in a range of frequencies. In the current investigation, lockin did not happen at $F_{R}=0.4$ but as will be seen the frequency of natural shedding was suppressed at the higher frequency ratios. However, at $F_{R}=0.4$, as will be seen in Figure 7(a), both the natural frequency and the oscillation frequency were present although the oscillation frequency was the peak frequency.

Figure 7(a) shows the power spectral density of the lift coefficient. Since the frequency of vortex shedding is same as the frequency of lift force fluctuations, the Fast Fourier Transform (FFT) analysis, which is performed to obtain the PSD plot, is performed on the time history of lift coefficient. As can be seen in Figure 7(a), the oscillation frequency which is 0.4 times $0.146 \mathrm{~Hz}$ (natural frequency from literature) dominates but does not suppress the natural frequency which is manifested as a slightly low value of $0.1332 \mathrm{~Hz}$. A similar pattern is seen in the PSD plots of $F_{R}=0.4$ at angular amplitudes of $10^{\circ}$ and $45^{\circ}$.

At the higher frequency ratio of 0.8 , there is suppression of the natural vortex shedding frequency. This is shown in Figure 7(b). The natural vortex shedding frequency is suppressed, and the shedding of vortices takes place at 0.8 times 0.146 , which is 0.1168 . PSD plots obtained for the other angular amplitudes $20^{\circ}$ and $45^{\circ}$ were also similar although the density value increased with increasing amplitude -787 and 866 for angular amplitudes $20^{\circ}$ and $45^{\circ}$ as opposed to 321 for $10^{\circ}$. The PSD plots of the lift coefficient, at $F_{R}=1.0$ and angular amplitudes $20^{\circ}$ and $45^{\circ}$ are shown in Figure 7(c) and Figure 7(d) respectively.

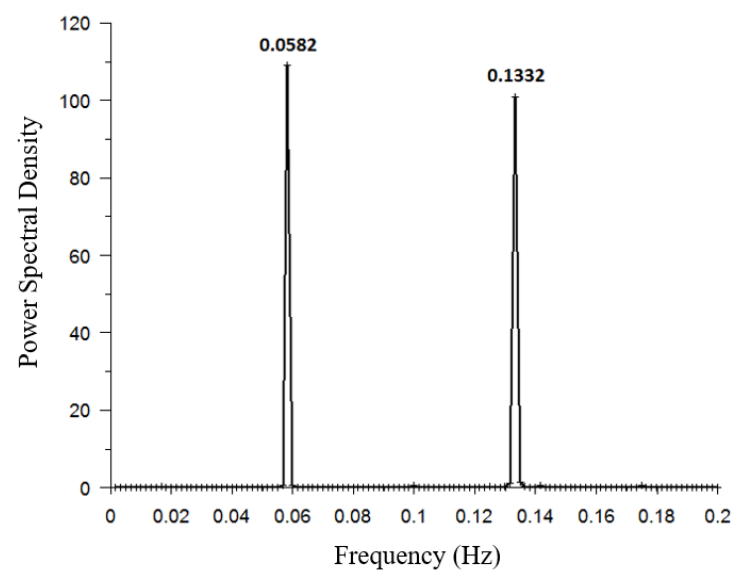

(a)

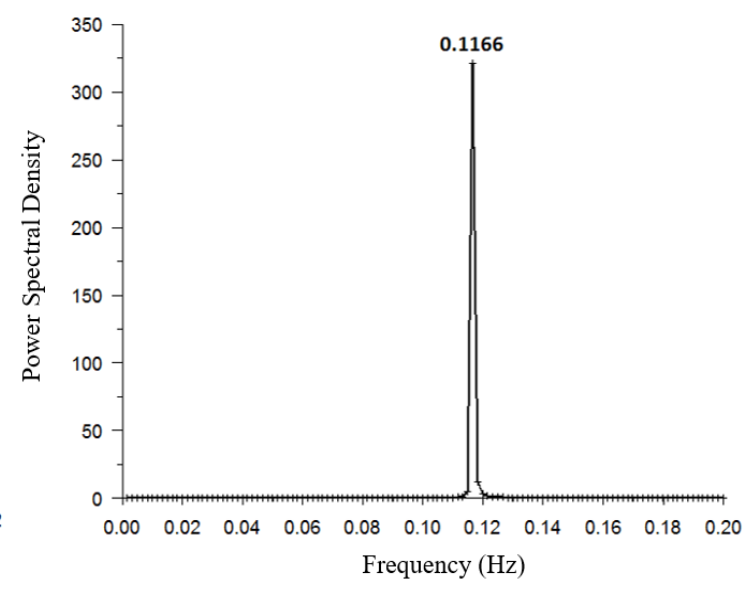

(b) 


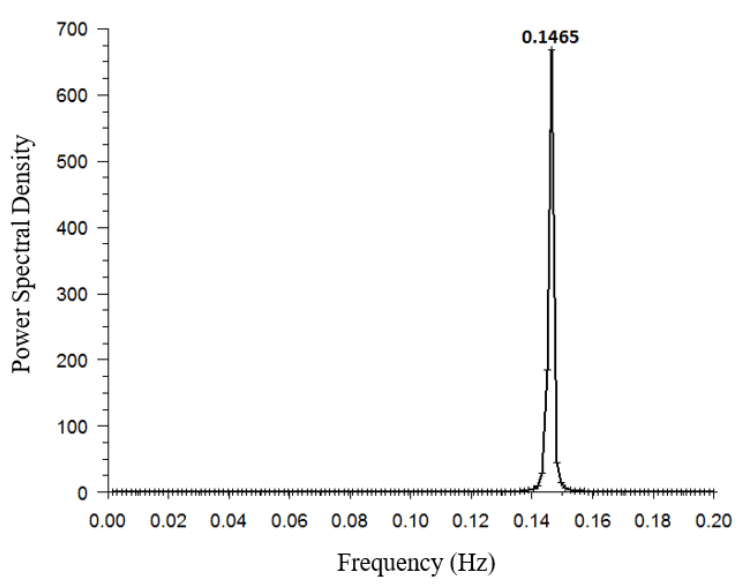

(c)

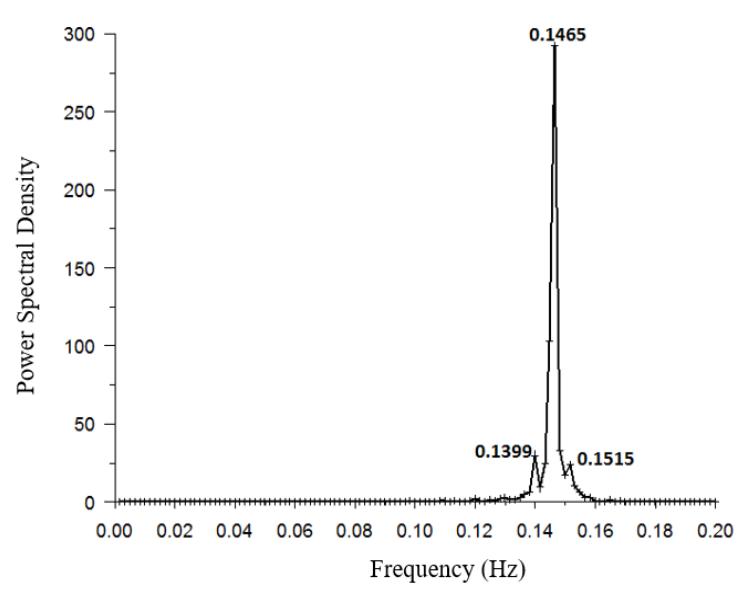

(d)

Figure 7. Power spectral density of lift coefficient at (a) $F_{R}=0.4, \theta_{0}=20^{\circ}$ (b) $F_{R}=0.8$,

$$
\theta_{0}=10^{\circ}, \text { (c) } F_{R}=1.0, \theta_{0}=20^{\circ} \text { and; (d) } F_{R}=1.0, \theta_{0}=45^{\circ} \text {. }
$$

From Figure 7(c) and Figure 7(d) it is evident that lock-in happens at $F_{R}=1.0$ at all amplitudes (PSD at $\theta_{0}=10^{\circ}$ is similar to that at $20^{\circ}$ ) although, at $45^{\circ}$, PSD seems to spread over a wider range of frequencies with local peaks. The presence of these local peaks is supposed to cause the periodic variations of the mean of drag coefficient and the fluctuation of lift coefficient with time at $\theta_{0}=45^{\circ}$ as shown in Figure 5.

\section{Flow Features in the Wake}

The width of a wake plays an important role in determining the pressure drag on the body, as it is related to the extent of the low-pressure region behind the body. The effect of angular amplitude on the width of the wake at $F_{R}=1.0$ is shown in Figure 8. In Figure 8, the wake width is denoted by ' $W_{w}$ '. As can be observed in Figure 8 , at $F_{R}=1.0$, wake width is altered by an increase in angular amplitude. Though it is evident from Figure 8 (c) that wake width increases, the change in wake width between angular amplitudes $10^{\circ}$ and $20^{\circ}$ is not prominent and it is within doubt whether there was an increase in wake width at $\theta_{0}=20^{\circ}$. This trend was seen at other frequency ratios as well. So, it is supposed that there could be a critical angular amplitude, beyond which a realisable increase in wake width occurs. All these inferences have been made base on visual observation.

The other important feature that is shown in Figure 8 is an estimate of how closely to the cylinder surface is a vortex shed. In Figure 8, this distance has been denoted by ' $S_{L}$ ' (for shedding length) and it is clear without doubt that the value of $S_{L}$ decreases with increase in angular amplitude although, at $\theta_{0}=45^{\circ}$, the reduction is drastic which takes us back to the previous hypothesis about the existence of a critical angular amplitude. Other common features such as the reduction in the vorticity magnitude of the shed vortices as they travel downstream are very much evident from Figure 8. 

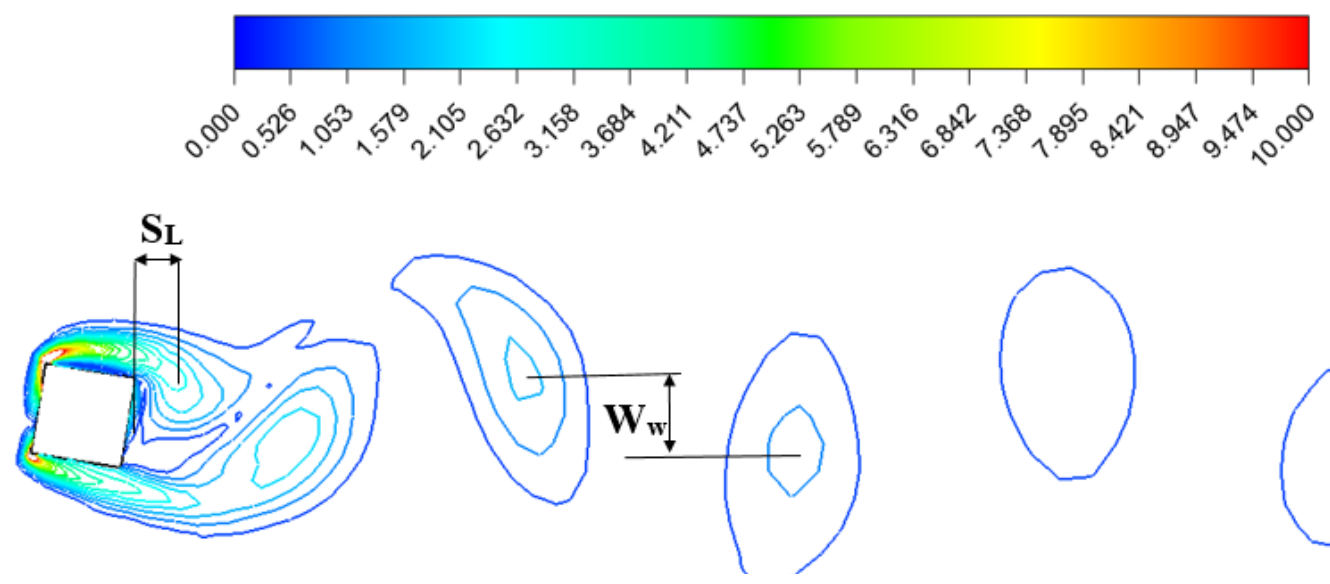

(a)
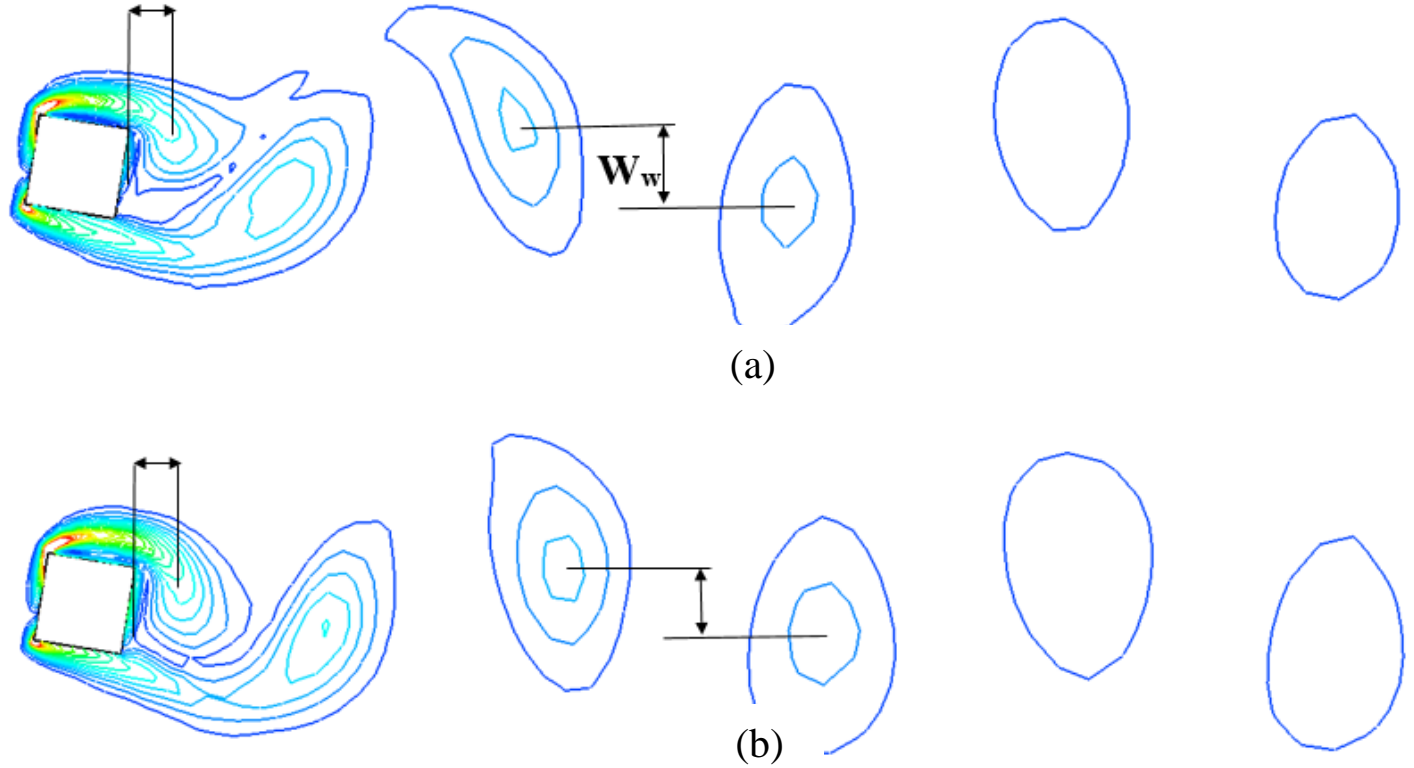

(b)
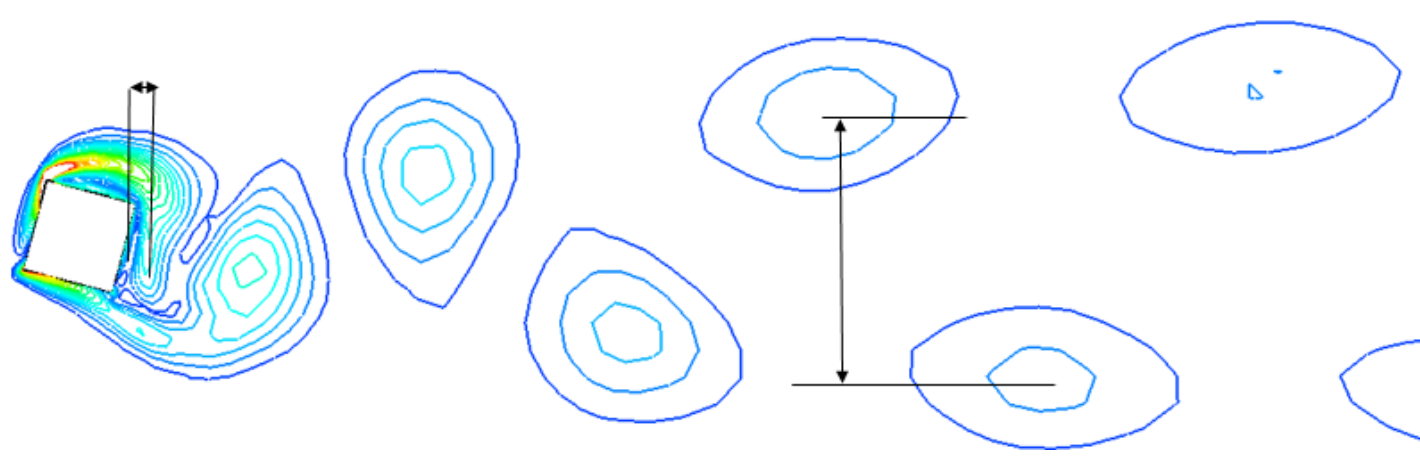

(c)

Figure 8. Vorticity contours and their features at $F_{R}=1.0$ at various angular amplitudes, $\mathrm{W}_{\mathrm{W}}$ - wake width, $\mathrm{S}_{\mathrm{L}}$ - shedding length of (a) $\theta_{0}=10^{\circ}$, (b) $\theta_{0}=20^{\circ}$, (c) $\theta_{0}=45^{\circ}$.

\section{Fluid Motion in the Vicinity of the Cylinder}

It is clear that the oscillating motion of the cylinder affects the flow, at least near its surface, but how exactly this manifest in the fluid motion was not clear and hence this was investigated as part of the present study. The case of $\theta_{0}=45^{\circ}$ at $F_{R}=1.0$ was chosen for this study. Two-time steps of the simulation were chosen such that at these two instants, the positions of the oscillating cylinder were nearly the same but the angular velocities of the cylinders at these positions were opposite in a sense. The instantaneous velocity vectors for the two instants, $568 \mathrm{~s}$ and $600 \mathrm{~s}$, are shown in Figure 9. 


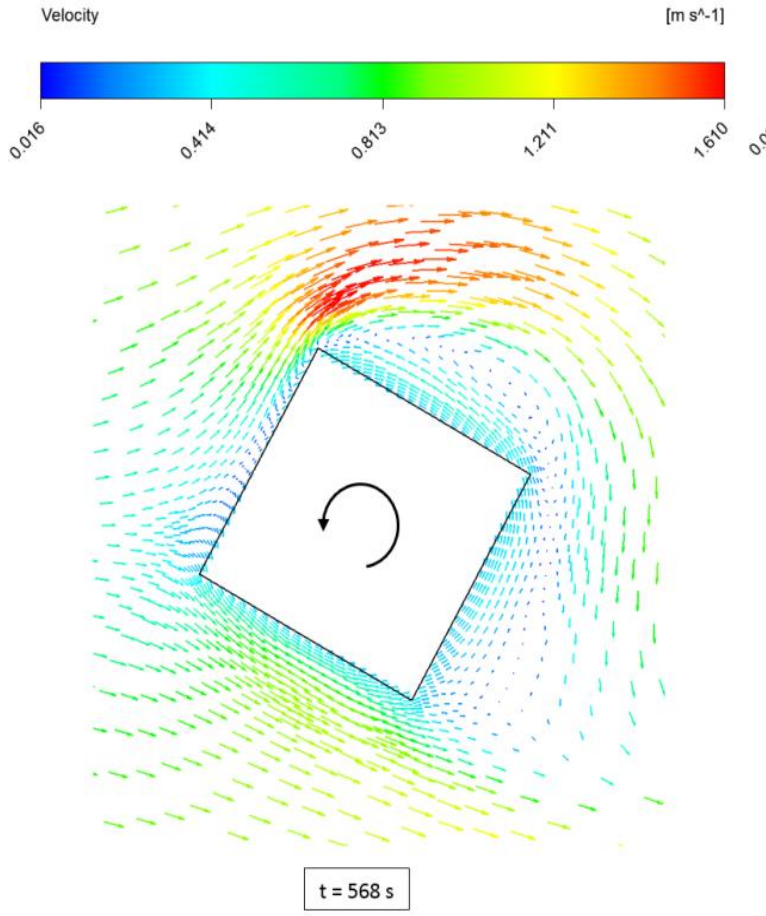

(a)
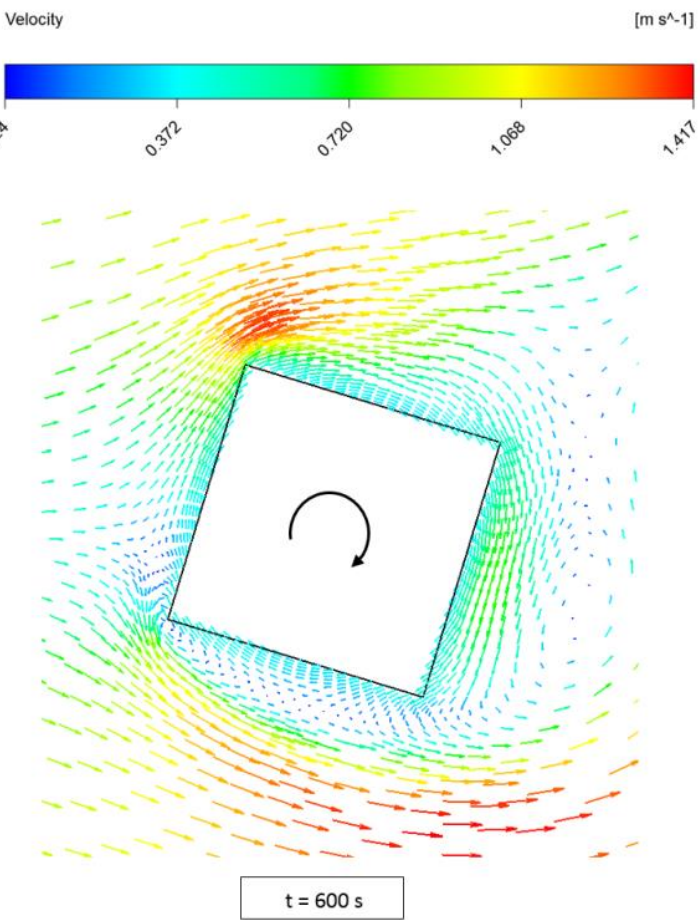

(b)

Figure 9. Velocity vectors near the cylinder surface at flow time (a) $t=568 \mathrm{~s}$ and;

$$
\text { (b) } \mathrm{t}=600 \mathrm{~s} ; \mathrm{F}_{\mathrm{R}}=1.0, \theta_{0}=45^{\circ}
$$

In Figure 9, it can be seen that due to the instantaneous motion of the cylinder in the counter-clockwise direction, there are layers of fluid above the cylinder that moves in the counter-clockwise direction. But a little far away above the cylinder, it can be seen that there is a deceleration in the flow of fluid. Below the cylinder, there is a slight acceleration of the fluid. This acceleration-deceleration effect was seen in the case of a stationary cylinder as well, and it could be explained in terms of the curvature effect. However, the effect of oscillation becomes clearer when we consider the vector plot at the other instantaneous flow time of $600 \mathrm{~s}$.

At the instant of flow time $600 \mathrm{~s}$, the angular velocity of the cylinder is in the clockwise direction. What is also obvious from the figure is that the acceleration which was observed in the region under the cylinder seems to have increased in this case. The reason for this was not apparent, but it is suggested that this could be due to the fluid being pushed down due to which there is an increase in velocity, an effect similar to what is found at the edge of a boundary in the flow over a flat plate, which is caused by the displacement thickness. There could also be a counter effect - a reduction in acceleration due to the motion of the fluid and the cylinder in the opposite directions under the cylinder at $\mathrm{t}=600 \mathrm{~s}$ but its extent was not investigated. Results from simulations done at $\mathrm{F}_{\mathrm{R}}=1.0$ and $\theta_{0}=20^{\circ}$ were also derived similarly and they are presented in Figure 10. 


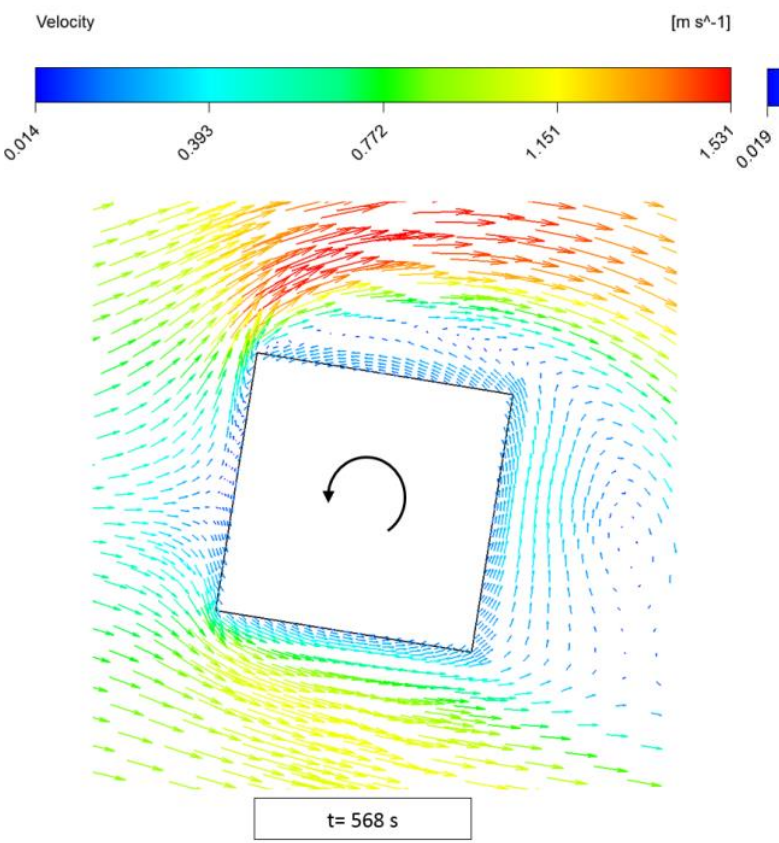

(a)

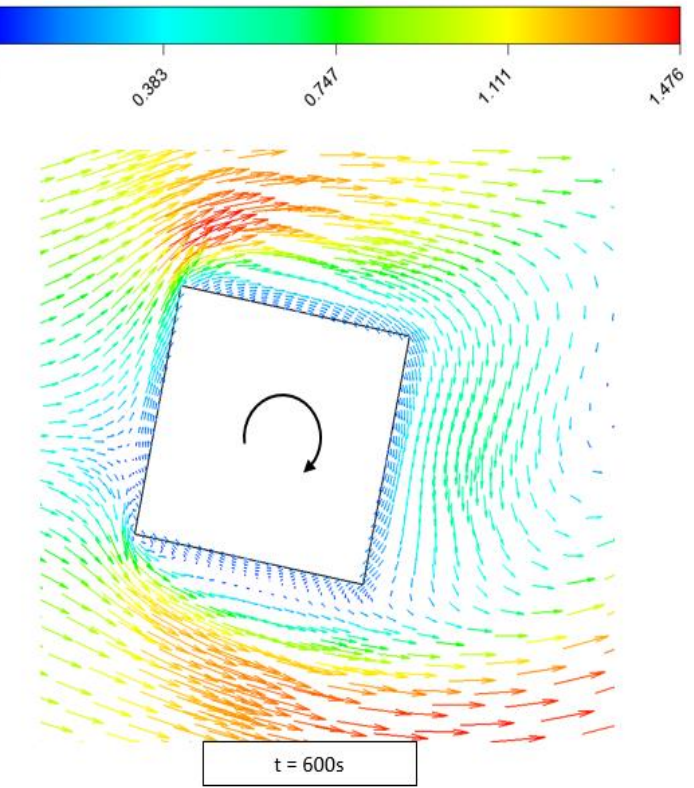

(b)

Figure 10. Velocity vectors at $F_{R}=1.0, \theta_{0}=20^{\circ}$ and instantaneous flow times of (a) $568 \mathrm{~s}$ and; (b) $600 \mathrm{~s}$.

In Figure 10, at $568 \mathrm{~s}$, it can be observed that below the cylinder, there is an acceleration followed by a slight deceleration of the fluid. This allows us to explain this phenomenon in the other way - the motion of the cylinder in the counter-clockwise direction reduces the acceleration of the fluid in the region below the cylinder.

\section{CONCLUSION}

The problem investigated and presented here is concerned with the simulation of uniform flow, past a square cylinder rotationally oscillating, at a Reynolds number of 100; the Reynolds number is based on the width of the square cross-section.

Two important observations from the investigation regarding the effect of oscillations on non-dimensional variables are as follows. First is the observation of a drastic reduction in the RMS value of the lift coefficient $\mathrm{C}_{\mathrm{L}, \mathrm{rms}}$ observed at the Frequency ratio of 1.0 when the angular amplitude is increased from $20^{\circ}$ to $45^{\circ}$. The other is the inference that the increase in angular amplitude has a greater impact on the drag coefficient at higher frequency ratios, which is evident from the drag coefficient plots.

Flow in the cylinder vicinity is no less important as the oscillations have been observed to cause an increase or a decrease in the fluid acceleration depending on the direction of the relative motion of the cylinder- when both move in the same direction, the acceleration of fluid decreases and when both move in opposite directions the acceleration increases. These observations were made in the region beneath the cylinder when it was tilted in the clockwise direction. Regarding the effect of amplitude on wake width, the possibility of the presence of a critical amplitude to coincide with the sudden rise in wake width is suggested. 


\section{REFERENCES}

[1] Sohankar A, Davidson L, Norberg C. and. Numerical simulation of unsteady flow around a square two-dimensional cylinder. In: Twelfth Australasian Fluid Mechanics Conference, Sydney, Australia, pp. 517-520; 1995.

[2] Vickery B. Fluctuating lift and drag on a long cylinder of square cross-section in a smooth and in a turbulent stream. Journal of Fluid Mechanics 1966; 25(3): 481494.

[3] Lee B. The effect of turbulence on the surface pressure field of a square prism. Journal of Fluid Mechanics 1975; 69(02): 263-282.

[4] Delany NK, Sorensen NE. Low-speed drag of cylinders of various shapes. NACA Technical Note 3038; 1953.

[5] Bearman P, Trueman D. An investigation of the flow around rectangular cylinders. Aeronautical Quarterly 1972; 23(03): 229-237.

[6] Taneda S. Visual observations of the flow past a circular cylinder performing a rotatory oscillation. Journal of the Physical Society of Japan 1978; 45(3): 10381043.

[7] Bearman P, Obasaju E. An experimental study of pressure fluctuations on fixed and oscillating square-section cylinders. Journal of Fluid Mechanics 1982; 119: 297-321.

[8] Roshko A. On the drag and shedding frequency of two-dimensional bluff bodies. NACA Technical Note 3169; 1954.

[9] Leontini JS, Jacono DL, Thompson MC. Wake states and frequency selection of a streamwise oscillating cylinder. Journal of Fluid Mechanics 2013, 730: 162-192.

[10] Zhao J, Jacono DL, Sheridan J, Hourigan K, Thompson MC. Experimental investigation of in-line flow-induced vibration of a rotating circular cylinder. Journal of Fluid Mechanics 2018, 847: 664-699.

[11] Kumar S, Lopez C, Probst O, Francisco G, Askari D, Yang Y. Flow past a rotationally oscillating cylinder. Journal of Fluid Mechanics 2013; 735: 307-346.

[12] Sohankar A, Norberg C, Davidson L. Low-Reynolds-number flow around a square cylinder at incidence: study of blockage, onset of vortex shedding and outlet boundary condition. International Journal for Numerical Methods in Fluids 1998; 26(1): 39-56.

[13] Orlanski I, A simple boundary condition for unbounded hyperbolic flows. Journal of Computational Physics 1976; 21(3): 251-269.

[14] Theory Guide, ANSYS Fluent Release 15.0, ANSYS Inc. November, 2013.

[15] Tu J, Yeoh GH, Liu C. Computational fluid dynamics: a practical approach. 2nd ed. Waltham: Butterworth-Heinemann; 2012. 